\section{Digital dental photography. Part 3: principles of digital photography}

I. Ahmad'

VERIFIABLE CPD PAPER
IN BRIEF

- We live in a digital world, and recent technological advances have offered conveniences and facilities that were once only stuff of dreams

- The eyes and digital sensors share uncanny similarities, unlike film photography that is rigid and inflexible

- Digital photography can be summarised by the acronym CPD (capture, processing and display).

Although we live in a digital age, our knowledge of the processes and technology involved is often limited. As a foundation to understanding the subsequent parts of this series, this part describes the fundamental aspects of digital photography, which includes the sensors, processing and display.

We are currently living in the digital revolution: digital broadcasting, digital consumer goods, digital dental radiography, and photography is no exception. Without doubt, digital is the future.

However, the natural world is analogue; everything around us is continuous: colour, space, time and sound are all sinuous, without discrete separations. We have separated nature, or digitised it, for the purpose of convenience, utilisation and manipulation. An example is time, which we have divided into days, hours, minutes and seconds, but which in reality, similarly to our surroundings, is not intermittent but

\section{FUNDAMENTALS OF DIGITAL DENTAL PHOTOGRAPHY}

1. Digital dental photography: an overview

2. Purposes and uses

3. Principles of digital photography

4. Choosing a camera and accessories

5. Lighting

6. Camera settings

7. Extra-oral set-ups

8. Intra-oral set-ups

9. Post-image capture processing

10. Printing, publishing and presentations

General Dental Practitioner, The Ridgeway Denta Surgery, 173 The Ridgeway, North Harrow, Middlesex, HA2 7DF

Correspondence to: Irfan Ahmad

Email: iahmadbds@aol.com

www.IrfanAhmadTRDS.co.uk

\section{Refereed Paper}

Accepted 15 November 2008

DOI: 10.1038/sj.bdj.2009.416

${ }^{\circledR}$ British Dental Journal 2009; 206: 517-523 passes continuously, without divisions or separations.

In a similar vein, digital photography offers many benefits compared to conventional photography including:

- Instantaneity and convenience

- Flexibility for editing, copying and disseminating images

- Environmentally greener by eliminating toxic dyes and processing chemicals

- Long-term economy by reusing storage media such as memory cards.

As with any new technology, there is, however, a learning curve to fully utilise the benefits and avoid the pitfalls. This chapter describes the fundamental aspects of digital photography, which serve as an essential foundation for subsequent chapters. The starting point is the quintessential item for digital photography: the sensors.

\section{THE SENSORS}

Light sensors can be categorised into three basic types, ocular, digital and chemical. Surprisingly, the fundamental principles of the three types are very similar. The ocular apparatus consists of the eyes, optic nerve and the brain, which is the ultimate arbitrator for assessment irrespective of the method used to create an image. In the light sensitive retina of the eyes, coloured dyes are stimulated by incoming light, triggering neural responses to the brain, which subsequently computes the image of the object being viewed.

In digital photography, light sensitive diodes act as the sensors, which create an electrical current, or charge, which is eventually processed into an image. Ocular and digital imagery share many similarities and are both extremely flexible. For example, if we see something we do not like, we can look away (with digital photography, unwanted parts of an image can be cropped). If something attracts our attention, the eyes concentrate on that specific part of the object or subject (with digital photography, any point of interest can be enlarged). Also, if we do not like what we see, the brain can change the context of reality so that we find the apparent unsightly representation more pleasurable (with digital photography, software manipulation can alter an image to any desired parameter). These few examples highlight the flexibility and uncanny similarities of ocular and digital imagery.

Conversely, chemical or film photography is rigid, with little scope for manipulation and therefore requires that all settings be exact if an acceptable image is to be produced. The basis of chemical photography is photosensitive coloured layers painted onto a film emulsion which, following development, reveals the registered image on a cellulose sheet. To produce a correctly exposed and high quality image, every setting needs to be accurate. For example, sharp focusing, correct orientation, proper framing and composition, precise aperture opening and shutter speeds. In addition, the colour temperature of the ambient light must match that of the film emulsion, and the developing chemicals need to be precisely diluted and at the correct working temperature. It is obvious 
that with so many variables, the scope of error is magnified and even if camera settings are correct, incorrect developing can produce unsatisfactory results. Besides the obvious convenience and instance of digital photography, a major advantage is the ability to correct technical errors at a later stage using software manipulation for rectifying exposure, white balance, framing, orientation, sharpening, etc.

A comparison of the three sensors, ocular, digital and chemical, is summarised in Table 1. It is worth noting that for chemical photography, the film sheet serves as the light sensitive sensor, storage and reproduction media. However, with the ocular and digital imagery, each of these three entities uses different media, which obviously expands possibilities for manipulation and offers unparalleled flexibility.

\section{TECHNICAL ASPECTS OF DIGITAL PHOTOGRAPHY}

The easiest way to describe the basic principles of digital photography is by dividing them into three processes, forming the acronym CPD:

- C for capture

- P for processing

- D for display.

\section{Capture}

The heart of all products based on silicone technology, such as computers, storage media, scanners and digital cameras, is a semiconductor. With image sensors, the semiconductors are photosensitive units composed of tiny light detecting units called pixels. The latter are a substitute for emulsion in conventional film cameras. Pixels come in many shapes and qualities, varying in size from $5 \mu \mathrm{m}$ to $12 \mu \mathrm{m}$.

Basically, the image sensor is a collection of silicone photodiodes (pixels), which register the intensity of brightness and darkness of an object. In effect, they are only capable of producing a black and white image of the object being photographed. To create a colour image requires using appropriate filters corresponding to the three additive primary colours red, green and blue.

Currently there are two types of image sensors competing in the market, the CCD (charged coupled device) and CMOS (complementary metal oxide semiconductors), each having advantages and disadvantages.

Table 1 Comparison of the three types of sensors
\begin{tabular}{l|l|l|l} 
& Ocular & Digital & Chemical \\
\hline Light sensitive sensor & Retina & Electrical diodes (pixels) & Dyes on film sheets \\
\hline Storage/relay media & Nerves & Memory cards or disc & Film sheet \\
\hline Reproduction media & Brain & Monitor, projector, print & Film sheet \\
\hline
\end{tabular}

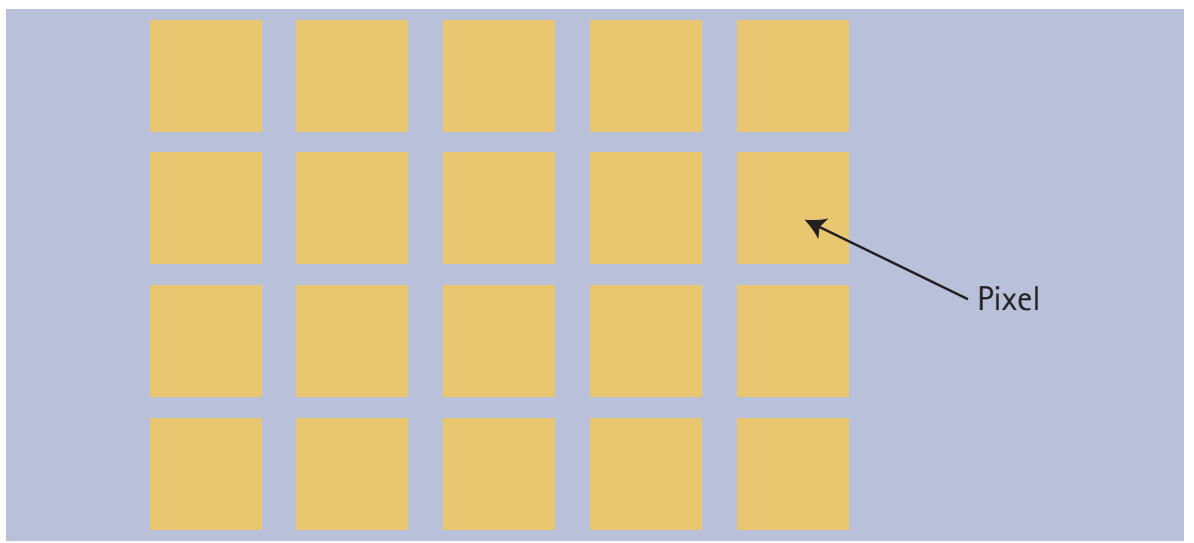

Fig. 1 Full-frame CCD with a large fill factor

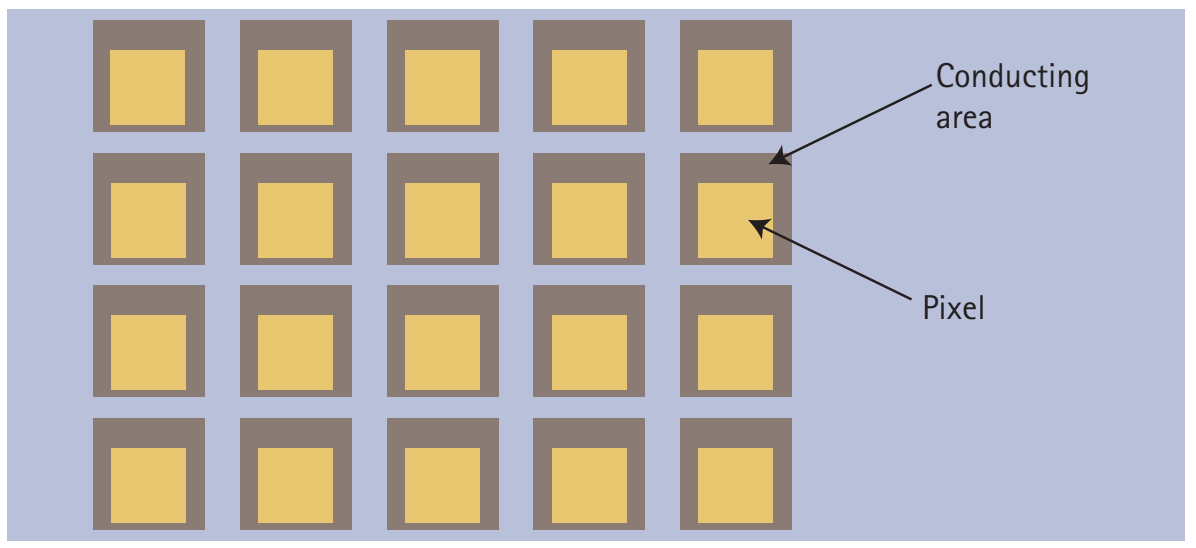

Fig. 2 Interline CCD with a small fill factor

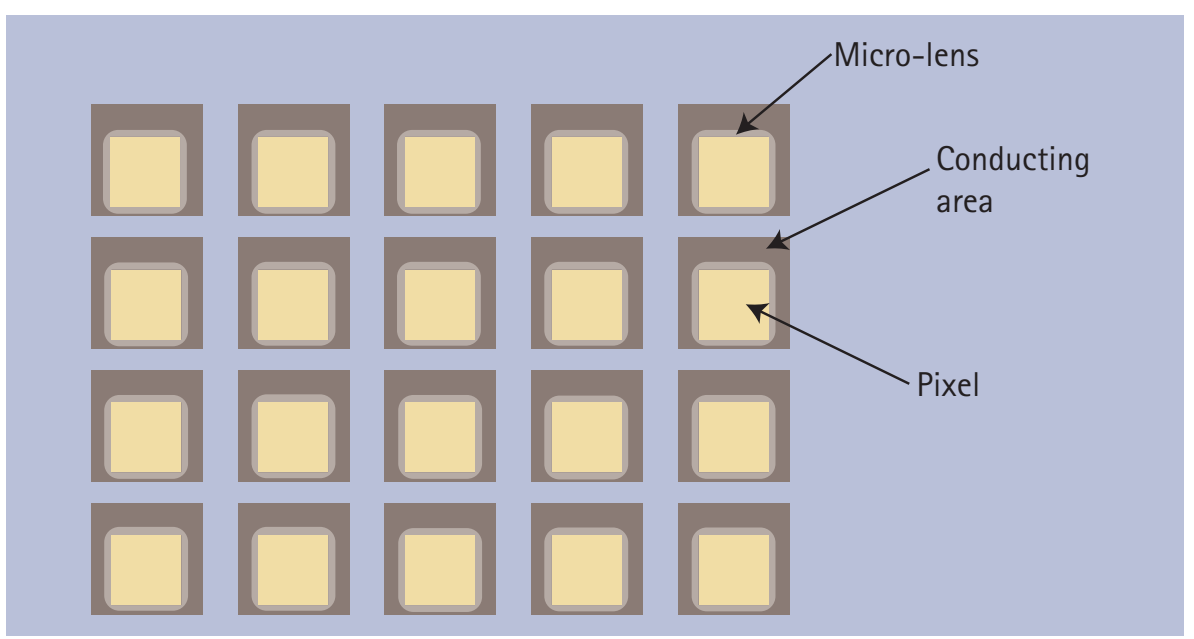

Fig. 3 Interline CCD with micro-lenses, which increase the fill factor

The CCDs can be further divided into fullframe and interline CCDs (Figs 1-2). The former, full-frame CCDs, allow the entire frame viewed in the viewfinder to be captured onto the sensor. The pixels are arranged in a line, and once stimulated by light, convey the electrical charge to the end of the line where it is processed 
to form an image. This procedure is time consuming and must be carried out in darkness, that is, after the camera shutter is closed. To expedite the process, the interline CCDs have non-light sensitive rows between the pixels which convey the electrical charge simultaneously as the pixels are 'stimulated' by the incoming light. This accelerates the process of creating an image, but due to the conducting row (non-light sensitive areas), the light sensitive area (fill factor) available is lower compared to the full-frame CCDs. This is a major advantage of full-frame CCDs, since a fill factor of $70 \%$ to $90 \%$ means that less image information is lost compared to an interline $\mathrm{CCD}$, which has a fill factor of $30 \%$ to $50 \%$.

The other competitors to CCDs are the CMOS sensors. These devices register light similarly to a CCD, but processing is performed on each pixel rather than being conducted to the end of a line. Due to the circuit integration within each pixel, the CMOS sensors have a smaller surface area with a reduced fill factor of only $30 \%$. Other limitations of CMOS sensors are a low dynamic range and increased noise levels, which are both detrimental to image quality. The advantages are lower power consumption and elimination of booming (overflow of excess electrical charges to adjacent pixels). To circumvent the low fill factor of both interline CCDs and CMOS sensors, either micro-lenses or octagonal shaped pixels with larger surface areas arranged diagonally are used to increase the light-sensitive potential or fill factor (Fig. 3).

\section{Processing}

There is a misconception equating an image sensor with digital images. However, the contrary is true. A sensor is only capable of delivering an analogue signal. This signal is an electrical charge, obtained from the result of exposing the pixels to light. Each pixel creates an electrical charge depending on the intensity of incoming light and the duration of exposure. Further technology is necessary to transform the analogue electrical signal into binary (digital) numbers. This is achieved by an analogue-digital converter (A/D converter). Once converted, the digital data is processed by microchips either within the camera, or downloaded to a PC and manipulated with appropriate

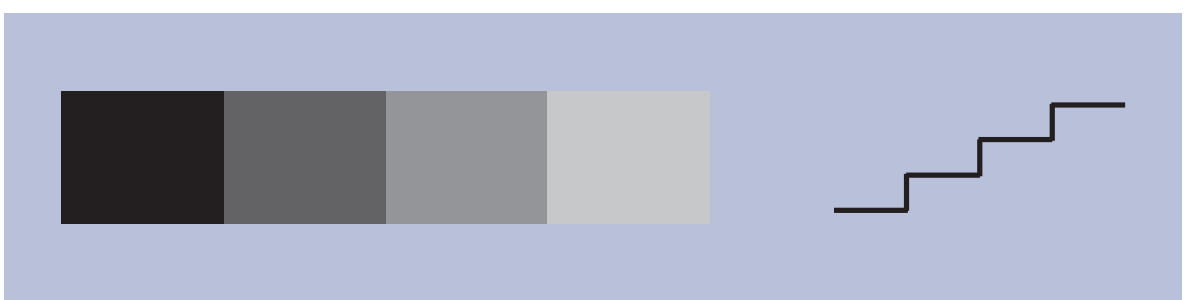

Fig. 4 A small bit depth results in a pronounced jagged edge

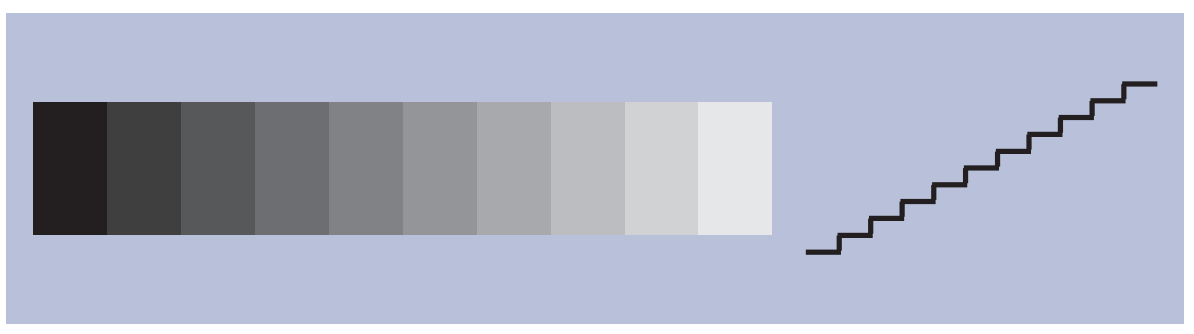

Fig. 5 Increasing the bit depth creates a smoother edge

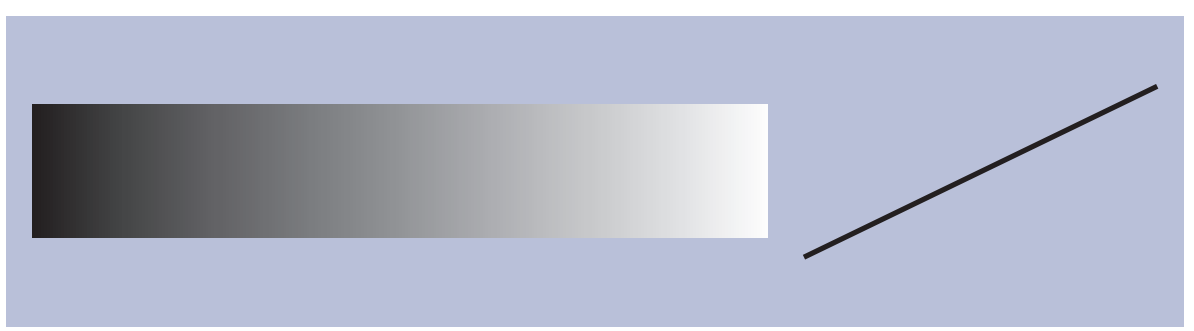

Fig. 6 To create a seamless transition between black and white and a smooth edge, a minimum bit depth of $\mathbf{8}$, or 256 levels, is necessary

software (for example, Adobe ${ }^{\circledast}$ Photoshop). Since all digital equipment and software work with a binary code, a transgression about digitisation is essential.

All computers function with a digital binary code, that is, they can only comprehend 0 and 1 . These binary digits are termed bits, and eight bits are equivalent to 1 byte. A byte is the minimum number of bits required to make up a single alphabetic character. The storage and memory capacities of computers are therefore quoted in megabytes (MB) or gigabytes (GB). In digital photography the primary function of the A/D converter is to reproduce the pure analogue signal into a digital code that is as close as possible to the original. Since digital data is composed of discrete entities, lacking homogeneity with noticeable banding, in order for the human eye to visualise a continuous tonal range (greyscale), a minimum of 8 bits is necessary $\left(2^{8}\right)$, which equate to 256 levels (Figs 4-6).

Consequently, for colour images comprising the three primary colours red, green and blue, each colour channel must have a minimum of 8 bits so that a continuous tonal range is perceived. This means 8 bit for red, 8 bit for green and 8 bit for blue (256 levels for red, 256 levels for green, and 256 levels for blue), or a total of 24 bits $\left(2^{24}\right)$. This is referred to as the bit or colour depth of an image. The greater the bit depth per primary colour, the greater the accuracy of recorded detail. The colour depth is an important point to consider when purchasing digital equipment or software. The stated colour depth can either be for each primary colour (per channel), or the total bit depth of the three primary colours. Many manufacturers quote 8-bit depth/channel, indicating 8 bit per primary colour (that is, 8 for red, 8 for green and 8 for blue), which equates to a total bit depth of 24. However, other manufacturers state the total bit depth of say, 24.

Returning to the image sensor, each pixel is assigned a binary number according to the magnitude of its charge. The A/D converter assigns the level of brightness in steps. As mentioned above, the greater the number of steps the smoother the transition on a greyscale and the more precise rendition of an image. Most cameras use an 8 bit per channel A/D converter coding for 256 different levels of brightness and darkness, while professional systems 
use 16 bit per channel, which translates to 65,536 brightness levels. To calculate the number of colours possible in a given system, the tonal levels for each colour are multiplied. For example, for a camera with a total colour depth of 24 , the number of colours coded is $256 \mathrm{R} \times 256 \mathrm{G}$ $\times 256 \mathrm{~B}$, which results in 16.7 million possible colours (Fig. 7), and for a total 48-bit system ( $16 \mathrm{bit} / \mathrm{channel}$ ) or $2^{48}$, the number of colours is $2.80^{14}$. In comparison, the difference threshold for colour of the human eye is low enough to discriminate 7 million colours.

In reality, only 8 bits are necessary for the eyes to visualise an uninterrupted smooth greyscale. However, once an 8-bit image is manipulated using photo editing software, there is degradation of the original 8-bit signal resulting in jagged steps at the periphery of objects. To avoid these unwanted artefacts, it is therefore wiser to start with a 16 bit image, allowing for degradation while still maintaining the minimum requisite 8 bit colour depth.

Besides colour depth, the other factor to consider is the dynamic range of the sensor (to be discussed further in Part 6). This is determined by the amount of charge that a pixel can accept, or its saturation level, termed full well capacity. The larger the physical size of a pixel, the greater the charge it can hold, and the greater the dynamic range. Therefore, in high-end digital cameras the sensors have larger pixels of approximately $12 \mu \mathrm{m}$, compared to the $5 \mu \mathrm{m}$ pixels that are used in amateur or compact cameras.

As previously stated, the image captured by a sensor is in essence black-and-white (Fig. 8). Colour is achieved by adding three channels representing the three primary colours of additive mixing, that is red, green and blue. A variety of ingenious methods are utilised for creating coloured images including rotating colour filters, beam splitters, or coating each pixel with filters of the three primary colours. The latter is the most popular method, creating a mosaic of red, green and blue on the image sensor (Fig. 9). When exposed, each pixel registers the intensity of light for one of the three colours, and when collated together this yields a colour image (Fig. 10). The latest technology is sandwiching three separate pixel layers of red, green, and blue, similar to the dye emulsions of film.

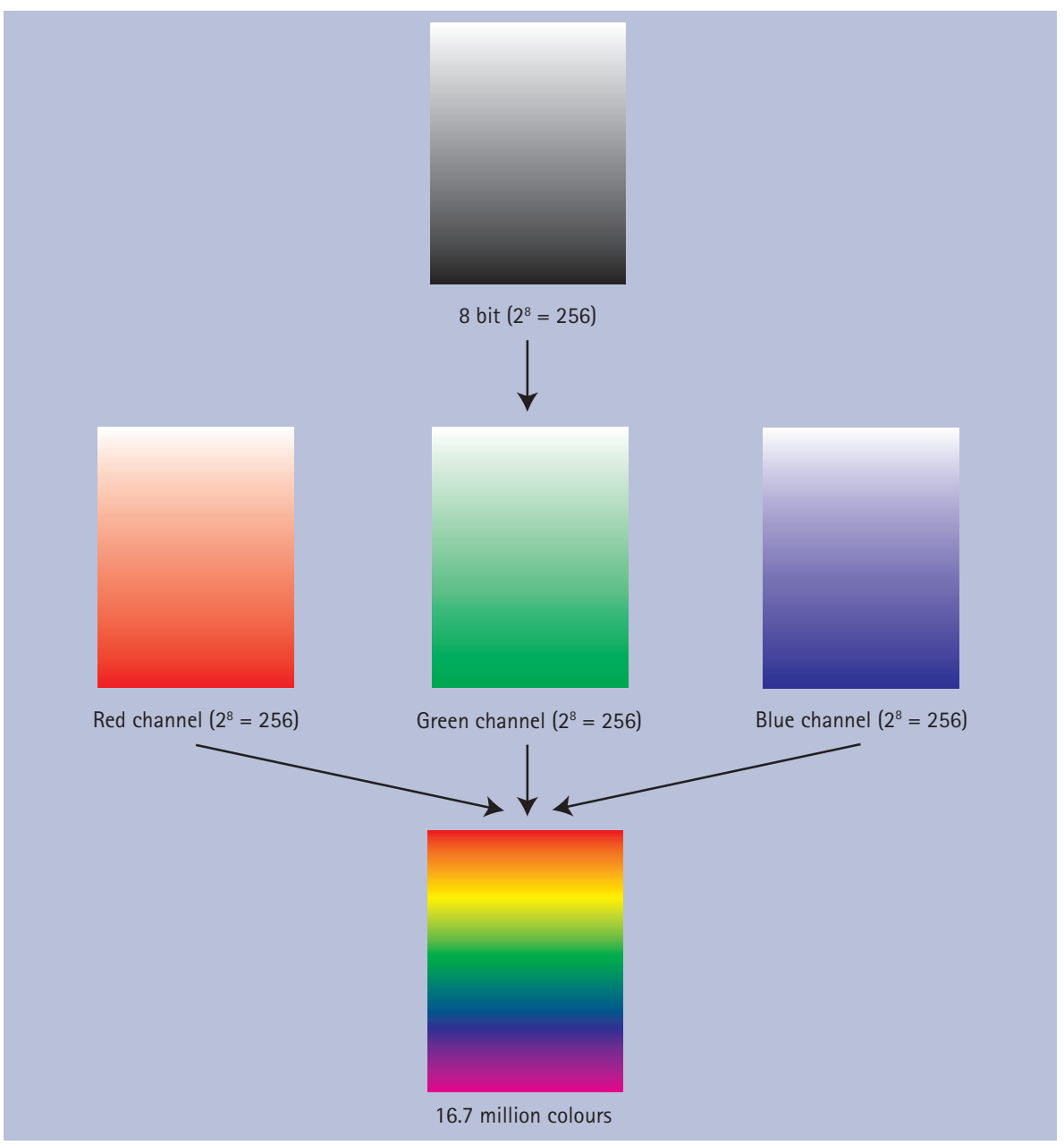

Fig. 7 Schematic representation of a 24 bit colour depth system (or 8 bit/channel)

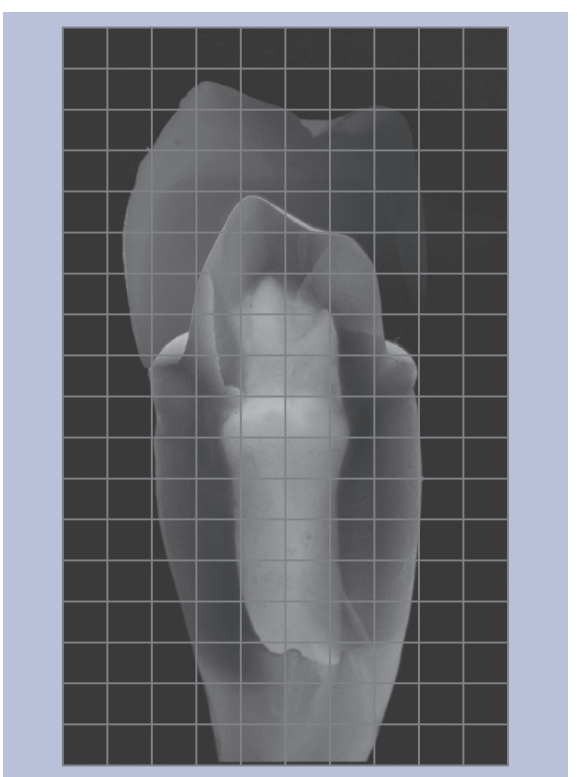

Fig. 8 The pixels of an image sensor only detect the brightness levels of an object, creating a black and white image

The rationale is that the red, green and blue components of white light penetrate at different depths: red is deepest, green intermediate and blue superficial. When these multi-layer sensors are exposed to

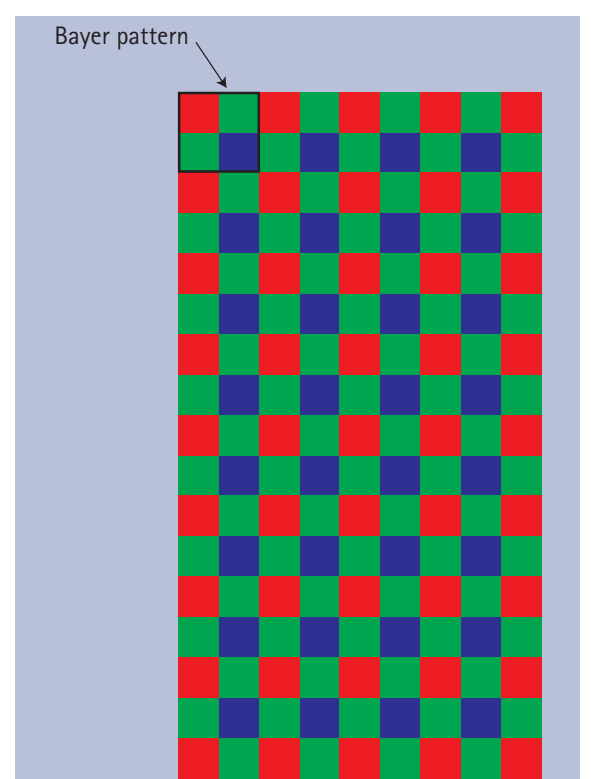

Fig. 9 Colour is captured by placing filters of red, green and blue onto the pixels, such as this Bayer pattern arrangement

white light, each individual layer registers red, green or blue, and when combined they form a coloured image.

The actual image capture is performed by numerous methods, for example scanning, 


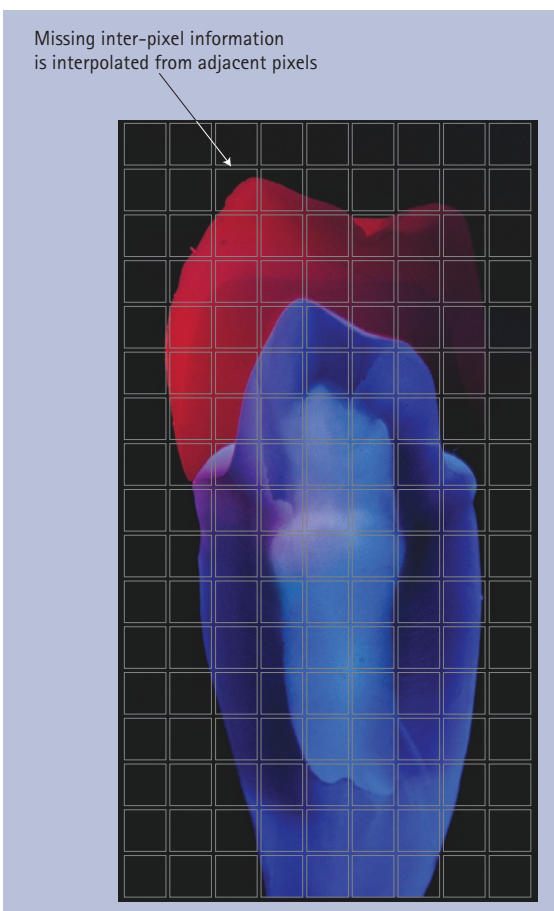

Fig. 10 The filtered pixels record the amount of red, green and blue at each site to form a colour image

3-shot, 1-shot, 4-shot, microscan and macroscan. The method used depends on the application in question. For example, for photographing static still-life compositions or documenting works of art and sculptures, the ideal is the scanner system. However, a scanning system is inappropriate for moving objects, and for sports photography the 1-shot system is the ideal choice.

By far the most popular system, and one that is suitable for dental photography, is the 1 -shot system. As the name implies, a single exposure is required to capture the object being photographed. The set-up is as follows. The image sensor consists of pixels with a mosaic of filters, for example in the Bayer pattern arrangement for the three primary colours, red, green and blue (Fig. 9). Once exposed, the sensor records the corresponding amount of red, green and blue in the prevailing composition. The entire process is summarised in Figs 11-15. Depending on the proximity of the pixels, small amounts of detail are lost, which are interpolated using information from adjacent pixels. The disadvantages of this system are that the resulting image is prone to interpolation errors such as colour fringes at edges of objects and Moiré patterns of strongly chequered materials. The interpolation algorithms mitigate these
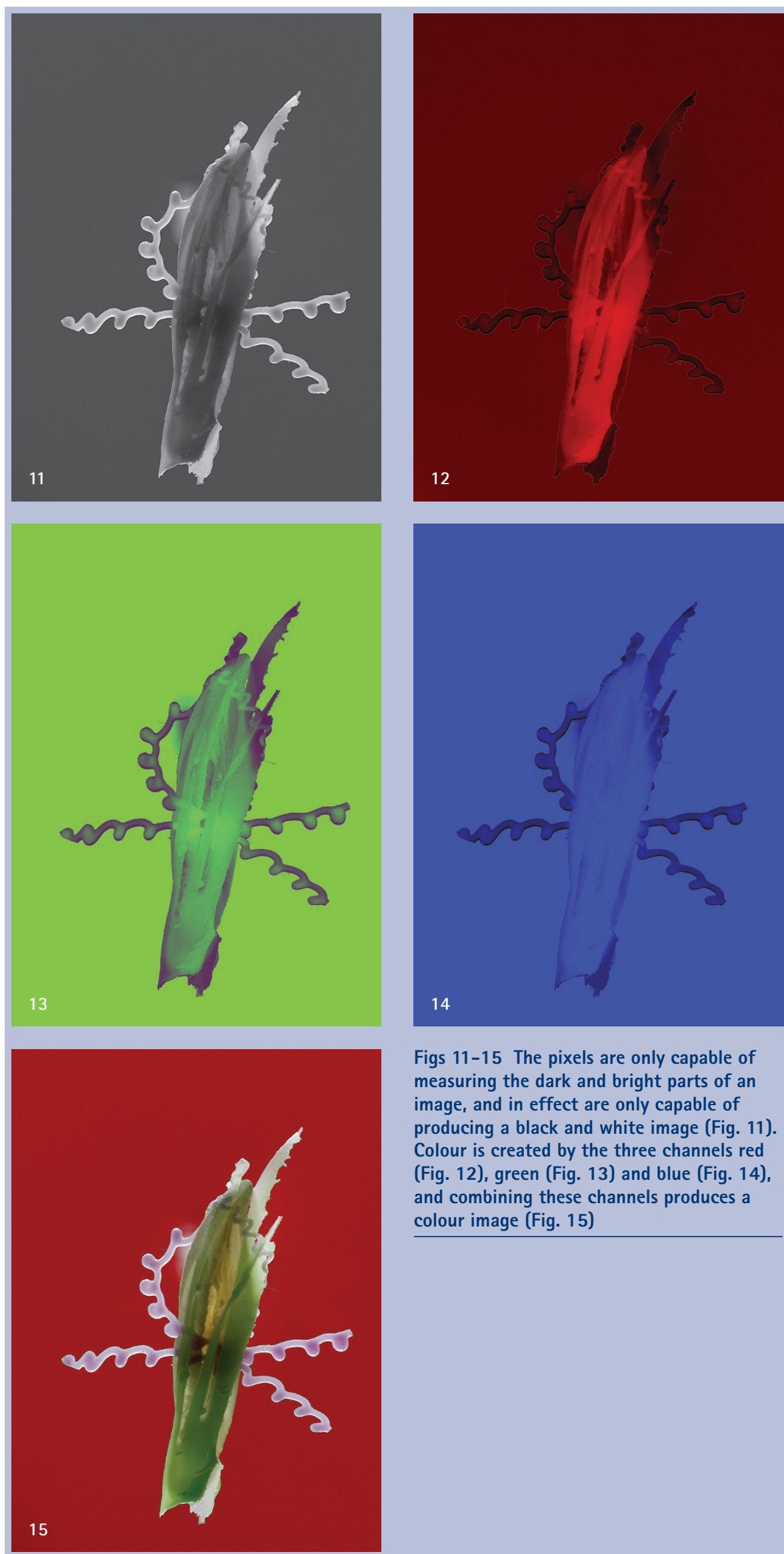

Figs 11-15 The pixels are only capable of measuring the dark and bright parts of an image, and in effect are only capable of producing a black and white image (Fig. 11). Colour is created by the three channels red (Fig. 12), green (Fig. 13) and blue (Fig. 14), and combining these channels produces a colour image (Fig. 15)

errors by using software to suppress these unwanted artefacts. However, if suppression is too great, genuine detail is lost, while a lengthy computation time may be unacceptable to expedite workflow. But taking all factors into consideration, the 


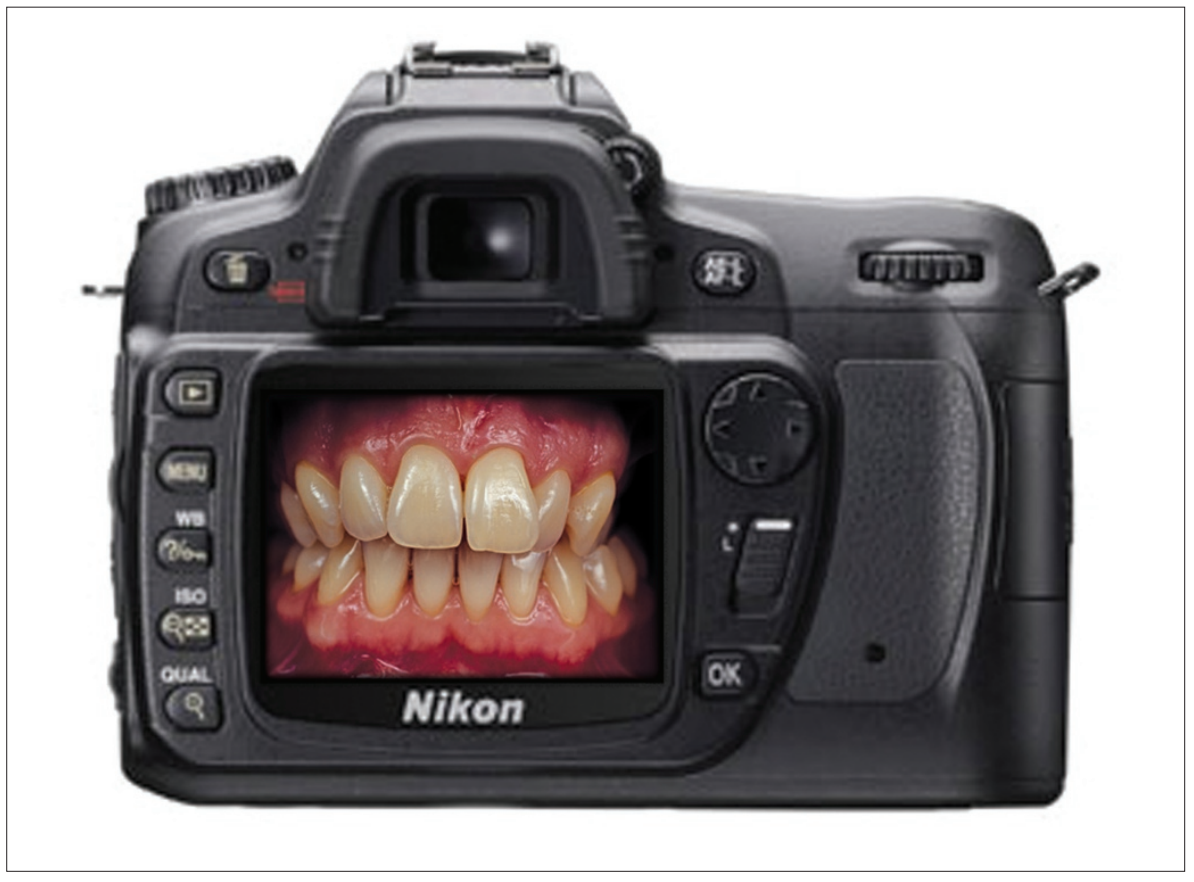

Fig. 16 LCD display on camera back

benefits of a 1-shot system, such as the ability to record moving subjects, compactness, lightweight and reduced cost, outweigh the minor and perhaps imperceptible loss in image quality.

Before an image can be viewed a certain amount of processing is necessary. Firstly, the captured image must be processed by software in the camera as a digital file. The format of the file at this stage can either be proprietary, that is specific to a particular camera manufacturer, or in a generic format such as RAW (PNG), TIFF or JPEG. Secondly, the size of the ensuing file depends on the format in which it is saved. The file size is a crucial determinant of the final image quality. The file size of an image can be calculated according to the formula:

Number of pixels $\times$ (total bit depth $\div$ 8) = image size in bytes

For example, the maximum file size that a digital camera with a 10 million pixel image sensor, and a bit depth of 24 (8 bits per primary colour) is capable of creating in an uncompressed state is $30 \mathrm{MB}$ :

$\left[10 \times 10^{6} \times(24 \div 8)\right]=30 \mathrm{MB}$

The file format, and hence its size, is primarily dependant on the intended use of the image. This is usually in a RAW or TIFF format. If a smaller size file is required, a low-resolution file format such as JPEG can be chosen. The latter format compresses the original digital file at the expense of detail loss, but is easier to store, manipulate and disseminate. The JPEG format also has a range of resolutions from low to high, with corresponding file sizes, respectively.

If a proprietary format is chosen, the file is in a raw state and requires processing by specific software before it can be viewed and stored into a generic format. On the other hand if a generic format is chosen at the outset, no further processing is necessary to view or store the file.

\section{Display}

After in-camera processing, the image can be displayed via electronic or printed media. Electronic media consists of monitors and projectors, and printed media of photographic paper or printing paper.

The first time that an image is usually viewed is on the LCD monitor on the camera back (Fig. 16) The size of these monitors varies from 2 inches to 3.5 inches, with a resolution ranging from $1 / 4$ million to 1 million pixels. The monitors allow instantaneous viewing of the image for assessing composition, framing, orientation and exposure. However, they are of little use for determining fine detail or sharp focus due to their low resolution, usually no more than 1 megapixels.

The second type of electronic viewing is with a computer monitor or an LCD projector (beamer). The resolution of both computer monitors and projectors varies enormously. For the former the resolution

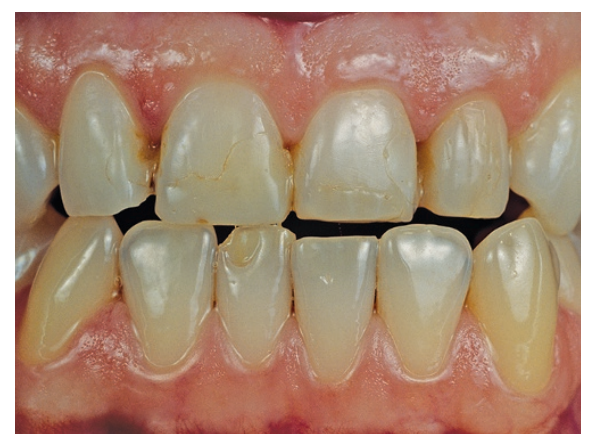

Fig. 17 An image with a file size of $30.3 \mathrm{MB}$ and $2,797 \times 1,895$ pixels (5.3 megapixels)

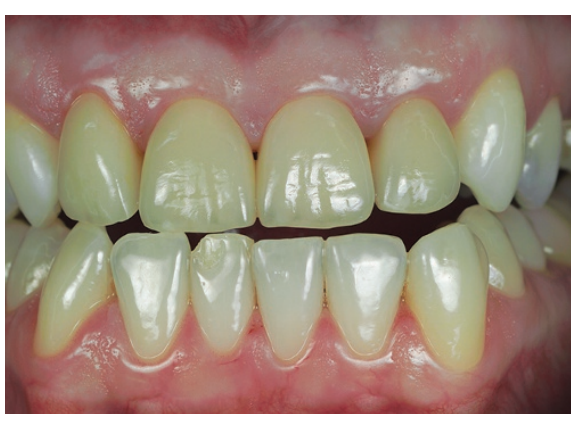

Fig. 18 An image with a file size of 113.4 MB and 5,329 × 3,717 pixels (19.8 megapixels)

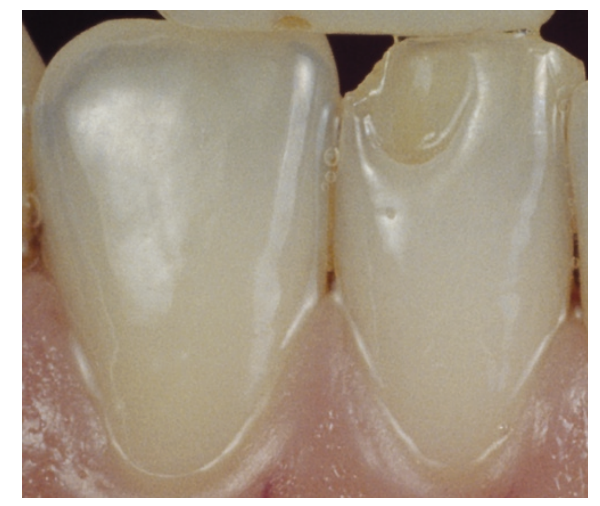

Fig. 19 A 100\% enlargement of a section of the image shown in Figure 17, with a file size of $2.3 \mathrm{MB}$ and $639 \times 616$ pixels (0.4 megapixels)

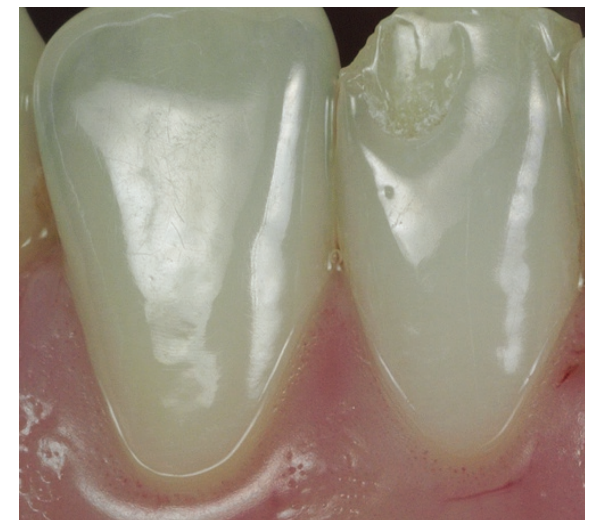

Fig. 20 A 100\% enlargement of a section of the image shown in Figure 18, with a file size of 7.6 MB and 1,140 × 1,153 pixels (1.3 megapixels) 
ranges from as little as $720 \times 480$ (0.3 megapixels) to $1,440 \times 900$ (1.3 megapixels). Even the state-of-the-art true high definition projectors are only capable of delivering a resolution of 2 megapixels $(1,920 \times$ $1,080)$, far short of what is achievable with even the most inexpensive digital compact cameras. This is the reason that an image taken with a 3 megapixels camera will look the same as that from a camera with a 10 megapixel sensor. If no difference is visually discernable, why bother with expensive, high megapixels cameras? The reason is as follows: the resultant image quality is not solely dependant on the number of pixels. Other more important factors include resolving power of the lens, tonal range of the entire system such as bit depth, dynamic range, file format and size, camera hardware (A/D converter, cooling), and image processing software (interpolation and colour reproduction), etc. Hence an elaborate camera system usually offers more than just higher megapixels, but also the features cited above to produce high quality images. This is an important point to remember before choosing a camera system (covered in Part 4), because two cameras with identical megapixels will produce drastically different quality images.
The final point worth mentioning about megapixels and monitors or projectors is as follows. While both have relatively low pixel counts compared to digital cameras, a difference is noticeable when an image is enlarged. For example, this is particularly relevant when photographing pathological changes to the oral mucosa. If a small lesion, in its early stages, is detected it is useful to magnify the area for detailed visual assessment. However, if the image deteriorates when enlarged, it is clinically useless, giving few clues to the pathological process. To illustrate this point, consider the two images in Figures 17 and 18 , which were taken with identical lighting, lens, etc. but with digital backs of different pixel count image sensors. The image in Figure 17 is a $30.3 \mathrm{MB}$ file, with a pixel count of 2,797 $\times 1,895$ (5.3 megapixels), while the image in Figure 18 is a 113.4 MB file with a pixel count of $5,329 \times 3,717$ (19.8 megapixels), that is, the second image has a nearly four times greater pixel count. When both images are viewed full-frame on a standard, 1.3 megapixel computer monitor, no difference is visually perceptible. The reason is that the pixel count of both images (5.3 megapixels and 19.8 megapixels) exceeds that of the monitor (1.3 megapixels). However, if both images are now enlarged by 100\% to concentrate on the lower mandibular incisors, the pixel count of the enlarged section of Figure 17 is $639 \times 616(0.4$ megapixels) - Figure 19, while for Figure 18 it is $1,140 \times 1,153$ (1.3 megapixels) Figure 20. With this enlargement, the pixel count of Figure 19 is lower (0.4 megapixels) than the monitor (1.3 megapixels), and the image appears grainy and is seen to be breaking down, with loss of detail. However, the enlarged image in Figure 20 matches the pixel count of the monitor (1.3 megapixels) and still appears sharp and retains detail. Notice the scratches on the enamel surface on the mandibular right lateral incisor in Figure 20, which are indiscernible in Figure 19. This example emphasises the need to use camera equipment with high specifications, including a high pixel count, to retain quality when an image is enlarged.

The final method of viewing an image is printing, which can either be with an office printer or a professional printing press. Both methods are ubiquitously popular, each having their unique benefits and drawbacks. Printing is discussed further in Part 10. 